\title{
MEDIACIÓN, EL DERECHO FUERA DE \\ LAS NORMAS: PARA UNA TEORÍA NO \\ NORMATIVA DEL CONFLICTO
}

Luis Alberto Warat 1

RESUMO: Este artigo refere-se à mediação como definição em aberto e no direito, como uma primeira aproximação de um procedimento sem regras de auto compositäo assistida dos vínalos conflitivos com o outro em suas diversas modalidades.

ABSTRACT: This article refers to the mediation like in open definition and in the Law, as one first approach as the unruly proceeding of self composition watched of conflictive link with the other in diverses aspects.

ÜBERSICHT: Dieser Aufsatz betrifft de Strcitbeilegung (Mediation) im allgemeinen und im Recht, als erste Amaherung an cin ungeregeltes Verfahren zur Herbeiführung cines Vergleichs durch die Streitparteien unter Beistand der Mediatoren, unter berücksichtigung der verschicdenen honflikterzeugenden Bindungen.

PALAVRAS-CHAVE: Metiação. Conflito. Composição.

KEY-WORDS: Mediation. Conflict. Composition.

SCMLUSSELWÖRTER: Sreitbeilegung (Mediation). Konflikt. Vergleic.

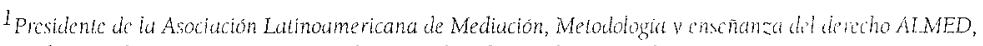
Proforor titular en vories programas de mestrado e doctorado on Brasil y Eiplika.
} 
1.1. Ouiero escribir este texto tratando de intervenir lo menos posible en los modos en que los juristas abordan el significado de la mediación. En un primer movimiento me propongo registrar una definición en abierto de la mediación que no sea una fuga de los marcos de referencia del imaginario común de los juristas. Por supuesto que no me privaré de introducir algunos elementos que direcciones las cuestiones para $\mathrm{mi}$ propuesta de considerar la mediación en termos de sensibilidad. En un segundo momento revistaremos las ideas juridicas sobre la mediación para situarla en el marco más amplio de las psicoterapias, el socio terapias, o las ecoterapias.

Entiendo la mediación en el derecho, en una primera aproximación como un procedimiento indisciplinado de auto-eco-composición asistida de los vínculos conflictivos con el otro en sus diversas modalidades.

1.2. Es un procedimiento, en la medida en que responde a determinados rituales, técnicas, principios y estrategias, que en nombre de la producción de un acuerdo intentan revisitar, psicosemioticamente, los conflictos para introducir una novedad en los mismos. Esa especie de movimiento enloquecido, impensado, impulsivo que muchas veces las personas realizan en media de encrucijadas de la vida, poblada de frustaciones que las conducen a situaciones crecientemente peores, debe ser sustituida (con ayuda del mediador) por una acción que incluya planes prácticos, antecipaciones, movimientos de sensibilidad, ideas de cómo actuar, siempre en la línea de una nova disposición para entender el mundo.

En el momento en que se menciona al acuerdo en mediación, sé esta escapando a la idea del acuerdo jurídico, se lo usa como una metáfora que, en el caso, evoca la necesidad de un compromiso interior que cada implicado en el conflicto debe realizar consigo mismo. Las promesas hechas para otros suelen hacerse para no ser cumplidas, no por mala fe sino por factores interiores que utilizan el discurso de una promesa como ilusión de un efecto performativo del lenguaje que las exime inconscientemente de cumplir, para ellos mismos, con lo prometido. Pensemos en las promesas de amor y en las garantías constitucionales elaboradas inconscientemente para no ser 
cumplidas. En ese orden de ideas el mediador tiene que ayudar a las partes a que acuerden con ellas mismas la necesidad de modificarse en el conflicto, que se prometan silenciosamente un cambio interior. Los sentimientos precisan, para ser incorporados como elementos transformadores de nuestra vida interior, del selencio. Las personas, por lo general, huyen de los silencios. Teatralizan los sentimientos para no sentirlos, se esconden en el escándalo de las palabras y en las promesas verbalizadas, que son otra forma de escandalizar para no hacer. El sentimiento sentido, hecho came, asumido por el cuerpo, el que nos hace es cambiar siempre aristocrático, precisa de la elegancia de los silencios.

Las cosas simples y vitales, como el amor, se entienden por los silencios que las expresan. La energia que puede estar dirigida a la rabia, a los celos, al dolor, tiene que sé hacer silencio. La persona queda serena en su silencio, brota en ella un sentimiento amoroso, de paz con ella misma llevando su destino para la autonomia. Esa es la mediación.

1.3. La mediación es, en segundo lugar indisciplinada por su heterodoxia, puesto que del mediador se exige que sepa moverse entre teorías, sin la obigación de defender un feudo intelectual o la ortodoxia de una capilla de clase o de saber. Al mediador le es dado pensar en el interior de un territorio abierto, con el descompromiso y la libertad propias del bricoleur, retiranco de los relatos de las partes lo que le resulta conveniente para lacilitar la auto-transformación del conflicto.

1.4. La auto composición de los procedimientos de mediación es asistida porque se necesita siempre la presencia de un tercero imparcial, aunque implicado, que ayude a las partes en su proceso de asumir los riesgos' de su autodecisión transformadora del conflicto. Lo que se busca con la mediación, que es un trabajo de reconstrucción simbólica, imaginaria e sensible de producción de dilerencias que permitan superar las divergencias, lo que exige siempre la presencia de un tercero que cumpla las funciones de un terapeuta emocional. 
1.5. El proceso es de autocomposicion en la medida en que son las mismas partes de un conflicto las que tratan de llegar a la produccion, con el otro adversario, de una diferencia que pueda recomponer, a través de una mimada interior, los ingredientes afectivos, jurídicos, patrimoniales o de otros tipos y generar, asi, lo nuevo en el conflicto.

El mediador tiene como función el ayudar a cada persona, envuelta en un conflicto, para que puedan aprovecharlo como oportunidad vital, un punto de apoyo para renacer, hablarse a sí mismo, reflexionar e impulsionar mecanismos interiores que los situen en una posición activa delante de sus problemas. El mediador estimula a cada miembro del conllicto para que encuentren, juntos, el rumbo que van a seguir salir de la encrucijada y recomenzar a andar por la vida con otra disposición. La actitud de búsqueda comun no debe perder de vista que deben tomar el conflicto como una oportunidad para administar mejor sus vidas, ir mas alla del probelma común y apostar en mejorar el proprio transcurso vital.

El trascurso vital es una permanente elección de caminos, con paradas temporales, las encrucijadas, donde miran los comienzos y se miden las consecuencias, mas o menos previsibles de cada elección. Ninguna elección garantiza el aciento, entretanto existen las estrategias inicialmente mas adecuadas.

Una encrucijada es una situación de duda vital, que modo diluso impulsa para el cambio de vida, elegir un camino vital entre varios posibles, muchas veces una elección dura que genera angustia y otros sentimientos dilíciles de administrar sin ayuda. Es necesario cambiar dentro de las propias necesidades vitales, a partir de la aceptación plena de nuestra existencia en el mundo, encontrarse con el doble (el otro dentro de nosotros) que se mueve escondido dentro de las proprias angustias, renacer de la melancolía como alternativa. Muchos somos victimas de nosotros mismos y debemos salir de esa situación, intentar un reaprendizaje de la vida. Siempre un reaprendizaje es preciso realizarlo con ayuda externa, psicólogo, mediador se precisa encontrar, con ayda, una posición activa para enfrentar una encrucijada; una disposición meditada, organizada, planificada en el diario vivir Una acción por la acción misma es un movimientro absurdo, una forma de irracionalidad que no conduce a nada productivo. 
Existen otras formas no judiciales de resolución de conflictos, como el arbitraje, en el cual el auto composición no incide en la misma medida debido a la presencia de un tercero que decide el conflicto. El auto composición relativa puede estar presente en el arbitraje, en los momentos en que él arbitra llama a las partes para una conciliación, o les solicita la colaboración conjunta en la reconstrucción del relato que necesita oír para tomar la decisión arbitral. Sin embargo, bajo ninguna circunstancia las partes, en el arbitraje, sé auto componen para decidir el conflicto. Las partes, a diferencia de los modos judiciales de resolución de las controversias, son escuchadas como gente. Eso ya es mucho como aspecto diferencial.

En la mediación el auto composición está referido a la toma de las decisiones. Se habla. De auto composición en la medida en que son las mismas partes comprometidas en el conflicto las que asumen el riesgo de las decisiones (o de las transformaciones del conflicto, conforme el caso). En el arbitraje el riesgo, por así llamarlo de la decisión corre por cuenta de los árbitros, en la misma forma en que ese riesgo es asumido por los magistrados en el momento en que deciden judicialmente un conflicto transformado en litigio.

En tomo de la mediación preliero hablar de autocomposicion, a pesar de que muchos autores prefieren optar por la expresión negociación que, como yo lo entiendo. En sus diversas modalidades transforma el proceso de mediación en un acterdo de intereses patrimoniales, en la medida en que la sensibilidad y los afectos no se acuerdan ni se ajecutan. Por otro lado, se puede considerar que, cuando se habla de negociación se hace referencia a un procedimento basado en propuestas explícitas e no en un trabajo de ayda sobre los no-dichos de un conflicto.

1.6. En la mediación la auto composición es ecológica, eco-autocomposición. Por dos fuertes razones La primera porque ella puede ser considerada como una forma de realización de la autonomía, en la medida en que educa, facilita y ayuda en la producción de las diferencias (prođucción del tiempo con el otro) que modifican las divergencias. La autonomia, como la democracia, o el amor, el odio, son formas de 
convivencia con la conllictividad, con la imposibilidad de ser completo que la conllictividad existencial determina. El individuo autonomo precisa negociar con el otro la producción conjunta de la diferencia, lo que implica, forzosamente, la mediación de lo simbólico. En segundo lugar, la mediación es una forma ecológica de auto composición en la medida en que al procurar una negociación transformadora de las divergencias, facilita una considerable mejoría en la calidad de vida.

1.7. Existen varias corrientes sobre el sentido, funciones y destino de la mediación. Ej punto de vista que yo adopto considera la mediación como un procedimiento radicalmente distinto de la conciliación y del arbitraje. La distinción se da por el carácter transformador de los sentimentos que, gracias a la mediación, pueden ocurrir en las relaciones sentimentalmente conflictivas; principalmente en lo que atane a la posibilidade - aprovechando positivamente el conflicto - de un encuentro consigo mismo.

La concllación y la negociación parecen tencr los mismos rasgos familiares, procedimentos que pueden incluirse en una misma clase, y hasta pensarse como variaciones semánticas de un mismo significante. Las diferencias, como yo encaro estos asuntos, saltan a la vista. La conciliación y la negociación no trabajan el conflicto, reduciéndolo a un enfrentamiento de intereses, en algunos casos salpicados por cuestiones emocionales. En ninguno de los dos supuestos el conflicto es translormado. El conciliador ejerce la lunción de negociador de litigios y por lo tanto no transforma $e l$ conflicto reduciendolo, sobre toda las relaciones afectivas y los vínculos de sensibilidad, a una mercadería. El fin de la conciliación está marcado por un acuerdo en que cada lado del conflicto promete renunciar a parte de sus pretenciones. Se acuerdan ciertas responsabilidades en el cumplimiento de algunas promesas ejecutables. Bajo ninguna circunstancia se trabaja una mirada interior que ayude a las personas a encontrarse consigo mismo, cambiar el rumbo de sus circunstancias, desarmarse de agresiones, o hacer la paz con su sombra (usando terminologia de Jung). En el fondo se trata de llegat a un acuerdo sobre elementos externos del conflicto (patrimonio u otro tipo de intereses) pero no se busca un acuerdo de sensibilidaci. La 
mediación no se preocupa con el litigio, porque no busca la interpretación normativa del conflicto, nia la verdad formal contenida en los autos del proceso. Tampoco tiene como finalidad aribar a un acuerdo negociado, se interesa por ayudar a las partes a redimensionar el conflicto, aquí entendido como conjunto de condiciones psicológicas, sensibles, culturales y sociales que determinan un choque de actitudes en el vinculo de las personas.

Algunos teóricos ce la mediación afirman que ella es un tipo particular: de negociación, una transacción transformadora. Particularmente no me gusta usar la palabra negociación como referencia semantica para la mediación. Cuando hablamos de mediación hablamos de terapia y no de negocios. No existe, a mí ver negociación en la mediación, ni mediación en los negocios, por lo menos en el marco de los catálogos teoréticos. Cuando la mediación es tratada como una forma de negociación, el procedimiento mediador trabaja las consecuencias de la relación conflictiva y no los malestares internos que generan el conflicto. No existe relación conllictiva si una de las partes tiene sus conflictos internos administrados. La mediación es una forma de terapia emocional, no un asunto de negocios.

Existen corrientes de mediadores de orientación acuerdista que consideran él o conflicto como un problema que tiene que ser resvuelto en los términos de un acuerdo. Estamos hablando de una propuesta de mediación que se fundamenta en la ideología y en individualismo posesivo. En termos acuerdistas, la mediación tiene como destino a construcción de una solución (que todos aceptan) para un conflicto concebido como problema. Para los acuerdistas los conflictos se solucionan redactando os convenios.

La orientación acuerdista se apoya, como fue dicho, en una ideología individualista posesiva; esa ideología piensa en una sociedad construida por inclividuos que luchan por la satisfacción individual de seus deseos y intereses. A insatisfacción en esa ideología es la de los interesses insatisfechos.

Existe otra comiente que podaramos chamar de transformadora: básicamente ella consiste en la visualización del conflicto como una oportunidad para el ofrecimiento a las partes de la posibilidad de una mejora 
en la cualidad de vida. Es una corriente ecológica e tambien psicológica del conflicto.

La corriente transformadora se aproxima de nuestra postura personal en términos de mediación. Con todo, en la Teoria Contradogmática prefiero hablar de producción psicoterapéutica de diferencias con el otro. Puesto esto, prefiriría decir que la contradogmática propone una nueva corriente mediadora que llamo de mediación alternativa o terapia del reencuentro.

1.8. La mediación es un procedimiento de intervernción sobre todo tipo de conflictos, termina, así, siendo mucho más que un instituto procesal. Para hablar de mediación tiene que introducir una teoría del conllicto más psicológica que jurídica. En el momento en que los juristas hablan de conflicto lo reducen a la figura del litigio, lo que no es lo mismo. Cuando se decide judicialmente, por medio de un litigio se considera normativamente los efectos; de este modo el conflicto puede quedar cristalizado, retomando agravado en cualquier momento luturo. Los juristas cuando interviene en un conflicto, apelan al imaginario jurídico, que yo denomino de sentido común teórico del Derecho.

En un litigio los jueces deciden lo pretendido por las partes conforme procedimientos de interpretación de las nomas y referencias dogmáticas, sin llevar en consideración lo querido y sentido por las partes. Presentar el conllicto como litigio implica no tener en cuenta la necesidad de trabajar en su devenir temporal. Los jueces operan sobre los contlictos interdictándolos, - congelandolos en el tiempo, eliminando la variable temporal para poder demarcar las controversins en un plano de abstracción juridica que permita controlar las variables con las que organizan sus decisiones. Los juristas, en la lógica del litigio, intervienen robándole tiempo mediante un proceso de anticipación idealizada del mismo, produce la anticipación del tiempo para provocar el efecto de un control normativo del futuro: simulan, para dar seguridad, que la ley puede controlar desde el presente los conflictos del luturo (eso gracias a uta mitica abstracción de la variable temporal). Producen una simulación del tiempo que impiden a las partes en contlicto elaborar sus diferencias al quedar sustraídas de su temporalidad. 
El concepto jurídico de conflicto como litigio representa una visión negativa del mismo. Los juristas piensan que el conflicto es algo que tiene que ser evitado. El sentido común teórico organiza su imaginario pensando el conflicto como controversial como disputa; una disputa, que por otro lado, se reduce a custiones dogmáticas, normativas e predominantemente patrimoniales. Los juristas nunca piensan el conflicto en términos de satisfacción e insatisfacción emocional o sensible. Falta en el Derecho una teoría del conflicto que nos muestre como el conflicto puede ser entendido como una forma de producción, con el otro, de una diferencia,inscrivir, gracias al conflicto la diferencia en el tiempo como producción de lo nuevo. El conflicto como una forma de inclusión del otro en la producción de lo nuevo: el conflicto como otridad que permite administrar con el otro diferente para producir la diferencia..

Propongo construir una teoría del conflicto que parta da distinción del diferente y de la diferencia. El primero es la natural y ultima instancia de la impenetrable presencia del otro como diferentes Dos personas diferentes pueden jumtas producir la diferencia, lo nuevo en el tiempo y en el conflicto, todo mediante un trabajo con relación a las cosas diferentes (lo divergente) que todo el mundo porta.

El conflicto abordado desde un punto de vista psicológico nos coloca delante de un enigma que le da impulsión. A auto-eco-composición asistida transforma el conflicto en la medida en que se abre a un proceso vivido como enigma. Actuando sobre el enigma las partes pueden transferir o transformar su realidad; esto es, produciendo con la otro la diferencia.

La mediación, basada en presupuestos psicológicos e psicoterapéuticos de basa en una teoría del conflicto que no lo ve como algo maligno. La mediación muestra el conflicto como una confrontación constructiva y revitalizadora. El conflicto como un potencial constructivo, una oportunidad vital. La vida como un devenir conflictivo que tiene que ser vitalmente gerenciado.

La teoría del conflicto adoptada sitúa a la mediación, en especial, como una semiótica de la otridad, que intenta interpretar el sentido del conflicto a partir del lugar del otro. Llegar al secreto del otro para decubrir 
los efectos internos de lo que afecta al otro, y con eso descubrir los propios. La mediación es así una forma alterativa (con el otro) de intervención en los conflictos. Hablar de alteridad es decir mas cosas que una simple referencia aun procedimiento cooperativo, solidario de mutua auto composición. Estamos hablando de una posibilidad de transformar el conflicto y de nosotros transformarnos en el conflicto, todo gracias a la posibilidad asistida de poder nos mirar a partir de la mirada del otro, colocarnos en el lugar del otro para entenderlo y entendernos. La doble mirada del otro. El dobre mirar en el otro. La mirada doblemente dirigida a la otra. Una mirada para el otro que permita llegar a su reserva salvaje a la nuestra (con el concepto de reserva salvaje quiero apuntar a todos los componentes amorosos y afectivos que ignoramos en nosotros mismos).

En fin, la alteridad, la otridad, como posibilidad de transformación del conflicto, produciendo en el mismo la diferencia con el otro. La otridad afecta los sentimientos, los deseos, o lado incosciente do conflicto, sin que exista la preocupación de hacer justicia o de ajustar el acuerdo a las disposiciones del derecho positivo. En este sentido, también habla en otridad o alteridad: a revalorización del otro del conflicto en detrimento del excesivo privilegio otorgado a los modos de decir el derecho en el litigio.

1.9. En las características precedentes fueron apareciendo otros aspectos que merecen ser comentados como complementos conceptuales de la primera aproximación a la idea de mediación. Hablamos que el procedimiento de la mediación se afectúa siempre en nombre del acuerdo, lo que no significa que el acuerdo sea primordial. Diferenciándose de los otros procedimientos de resolución no adversarial la mediación no tiene como meta llegar a un acuerdo. A mi entender, como vimos la función primordial de la mediación es la producción de la diferencia, instalando lo nuevo en la temporalidad conflictiva. A lo sumo es una terapia do reencuentro en la que se pueden negociar deseos y afectos, como acuerdos del corazón, acuerdos no ejecutables. Un sentido de negociación bien diferente al que puede presentarse en términos de conciliación. 
Destaco la mediación como un procedimiento psicoterapéutico alterativo (con el otro) de revisitacion de los conflictos con el propósito de ayudar a las partes a recuperar su energía vital y se encuentren en armonía con ellas mismas, para conseguir, en un segundo momento, modos de armonizarse con el otro y con el mundo. Seria un trabajo de sensibilidad de base semiotica, instrumentado principalmente a partir de jugos, tareas grupales y teatralizaciones.

Ta base semiótica de la mediación no lleva a considerarla como un trabajo de interpretación, que con ayuda de un tercero imparcial e implicado trabaja secretos de lo que fue enunciado para producir, en una inscripción simbólica con el otro, una diferencia en la historia del conflicto. De este modo estoy introduciéndola idea de la interpretación, en la mediación, con algunas tonalidades diferenciadoras de otros usos interpretativos. Aquí pretenco hacer referencia a la producción conjunta de una diferencia simbolica, lejos de cualquier tentativa de dominación. Hablo de la interpretación conjunta de un secreto (no de una falta). Una translerencia o desplazamiento semiotico del lugar del conflicto para el lugar de una oportunidad vital; la encrucijada.

1.10. La mediación también se caracteriza por la presencia de un tercero imparcial que ayuda; esto es, un sujeto que únicamente tiene poder de ayuda, no tiene poder para decidir el conflicto. El poder del mediador es para crear espacios transaccionales: un entrenostros afectivo-informativo que facilita a las partes a tomar decisiones. La función del mediador no es la función del poder decisorio, es un discurso amoroso destinado a inscribir las pulsiones en el registro de Eros.

No tiene mucho sentido, como la entervención del mediador es amorosa, hablar de imparcialidad de un modo parecido al modo en que se reclama la imparcialidad de un juez. El mediador no decide, no impone su criterio, no teniendo entonces ninguna sentido discutir su imparcialidad. El mediador llama para el lugar de la transferencia, invita a las partes a que se reencuentren en sus pulsiones de vida, se transformen vitalmente. El deber del mediador es mas bien del orden de la abstinencia. 
El mediador tiene que retirar el conflicto del espacio negro de las pulsiones destructivas. Un territorio del que no escapa el Derecho, sus normas e procedimientos de sanción y venganza. El mediador tiene que trata de efectivizar el conflicto inscribiendo el amor en medio de las pulsiones destructivas, el amor en medio del poder, en medio de la dependencia y de la dominación.

El mediador ayuda a las personas a comprender sus conflictos con mayor serenidad y sensibilidad, retirando de ellas la carga de energía negativa que impide su administración serena. Es a través de una lectura corporal y de un trabajo de florecimiento de la sensibilidad, porque muchas veces las palabras no expresan el deseo de las partes, que se pretende administrar de un modo más productivo los aspectos negativos del ego y de la racionalidad. Es decir un medidor que trata de despertar el potencial de buena convivencia con nuestros conflictos internos, para que se pueda redimensionar y mismo evitar los conflictos Inter-personales pasibles de procedimientos de mediación.

2.1. La mediación puede ocuparse de cualquier tipo de conflicto: comunitario, ecológico, empresarial, escolar, familiar, penal, político, de menores en situación de riesgo, del trabajo y del consumo; como también los de la realización de los derechos Humanos y de la ciudadanía.

No es absurdo pensar la mediación dentro de los conflictos del saber, considerarla una visión de mundo, un paradigma ecológico y un criterio epistémico de sentido. La mediación como la mejor formula hasta ahora encontrada para superar el imaginario del normativismo jurídico (una hipotesis fuerte de mi discurso, es decir, del modo en que mi Toría Contradogmática del Derecho y de la Sociedad encara la cuestión de la mediación).

En términos de autonomia, de ciudadania, de democracia y de derechos humanos la mediación puede ser vista como su mejor forma de realización. Las practicas sociales y políticas de la mediación se conliguran en un instrumento de ejercicio de la ciudadanía en la medida en que educan, 
facilitan y ayudan a producir las diferencias y a realizar tomadas de decisiones sin la intervención de terceros que decidan por los afectados en un conflicto. Hablar de autonomía, de democracia e de ciudadanía, en un cierto sentido, es ocuparse de la capacidad de las personas para sé autodeterminar em relación con los otros; auto determinarse, insisto, en la producción de la diferencia. La autonomía como una forma de producción de diferencias y tomada de decisiones con relación a la conflictividad que nos determina y configura en términos de identidad y de ciudadanía, un tabajo de reconstrucción simbólica y de politicas de acción en los procesos conflictivos de las diferencias que nos permite formar identidades culturales, - integramos en el conflicto con el otro -, con un sentimiento de pertenncia común. Una forma de poder percibir la responsabilidad que toca a cada uno en los conllictos, generando devenires reparadores y transformadores.

La autonomía, la democracia y la ciudadanía, como el amor, el odio y el dolor son formas de convivencia con la conflictividad y la indeterminación de la plenitud. Ninguno de los términos de la primera trilogía deben ser pensadas con relación a algo idealizadamente presentado como entero, como pleno, mas con relación a algo que nunca se cierra, que se constituye en relación con el otro, debiendo ser objeto de una permanente mediación. Pensar todos esos conceptos como si fuesen significativamente plenos es ideológico y alienante.

2.2. La mediación ultrapasa con creces la dimensión de la resolución no adversarial de disputas. Ella contiene incidencias y provoca efectos que son ecológicamente significativos como estrategia educativa, como realización de una política de la ciudadania de los derechos humanos y de la democracia, produciendo un devenir de subjtividades que indican una posibilidad de luga de la alienación.

La mediación debe ser encarada como una actitud general delante de la vida, como una visión de mundo, un paradigma ecológico y un criterio epistémico de sentido. De un modo general, la mediación puede ser vista como un componente estructurante da visión ecológica del mundo, un 
componente estructural do paradigma politico $e$ juridico da transmodemidad.

La mediación seria un salto cualitativo para superar la condicion juridica de la modemidad, basada en el litigio y apoyada en un objetivo idealizado e ficticio como es el de descubrir la verdad que no es otra cosa que la implementación de la ciencia como argumento persuasivo; una verdad que debe ser descubierta por un juez que puede llegar a pensar a sí mismo como un semidiós, sin dependencias o adicciones, en el descubrimiento de la verdad, que es solo imaginaria.

2.3. En la mediación es fundamental trabajar los no dichos del sentido, ellos revelan mucho mas del conflicto que lo expresado. No podemos olvidar que mediación se realiza siempre por la percepción y el trabajo realizado sobre infinitos detalles. Toda mediación, copiando a Clarice Lispector, esa espléndida poetiza brasileira, es hecha de infinitas paciencias y detalles que tiene que ser cuidados.

Occidente y su condición moderna nos cegó para el amor y para nuestra propria naturaleza interior. Nuestro inconsciente amoroso quedó impenetrable, lejos de nosotros mismos, rodeado de palabras que nos armarán contra nuestra propia alma. El mundo convertido en el lugar de los grandes simulacros, donde se multiplican al infinito las frustaciones, los dolores, donde la violencia y el odio hierven despacio por detrás de una apariencia de conformidad. Vivimos en sociedades donde los resultados, el exito personal, las armaduras con las que construimos nuestra imagen nos van alejando, de ensimulacro, de lo auténticamente sentimos.

Terminamos identificandonos con las ideas y los pensamientos, dejando de ir a los sentimientos. E más, cuando decimos lo que sentimos, en el fondo pensamos el sentimiento. Nuestra mente resuelve las cosas que amamos o odiamos, nuestra mente nos sitúa en los conflictos. Perdemos las posibilidades de escuchar al propio cuerpo cuando siente. 
2.4. La mediación, retomando para linalizar las aproximaciones, no seria otra cosa que la realización con el otro de los propios sentimientos. Mediar, como un modo de vivir, vivir en armonía con la propia interioridad e con los otros. La mediación como una posibilidad de tener el derecho a decir to que me pasa. Una búsqueda del propio punto de equilibrio y del punto de equilibrio con los otros. Un punto de equilibrio entre los sentimientos y las razones para evitar los sentimientos desmedidos. Una posibilidad de producir con el otro el entre-nos de la sensibilidad.

Las ideas, al contrario de los efectos de la sensibilidad, pueden mostrar falsificadamente los sentimientos, Cuando pensamos establecemos necesidades simbólicas que son en muchos casos falsas con relación a nuestro corazón, palabras exiladas del corazón. Este desdobramiento de la personalidad es, desde la perspectiva de los sentimientos, neurosis.

La mediación, en suma, no es una ciencia que puede ser explicada, ella es un arte que tiene que ser experimentado. Muchas escuelas de mediación piensan que forman mediadores como si fuesen magos que pueden calmar las partes con sus trucos. La magia es otra, consiste en entender a la gente, ser especialista en gente.

La sensibilidad es el gran tema del Derecho en la virada del milenio Otro gran tema es la mediación. Juntarlos es un modo de pensar el lugar del Derecho en la cultura emergente del tercer milenio. Extendiendo la idea diría que estamos hablando de una conjunción que detrmina la emergencia del paradigma ecológico; es decir, la orden de configuración de las instancias de la sociedade emergente, otra condición del estado, de la política, del Derecho, del amor, de la educación, del saber, de las verdades, de la sensibilidad y de los vínculos nacionales e internacionales.

Creo que el hilo conductor para escribir este esbozo de tópicos sobre mediación, fue el amor. Saber situar el amor en nuestra vida y saber situarnos en el amor requiere un trabajo de autoconocimento y maduración personal, para no confundirlo con la dominación la anulación personal, el sometimiento o cualquier forma de adicción. Entender el amor como un arte de vivir y de aprovechar las oportunidades vilales es un buen indicador de aprovechamiento de los conflictos a favor de un mayor conocimiento de si mismo. 
Me preocupe, asimismo, por apreciar el origen sensibile del pensamento y de la acción, sus raíces en el encuentro con la alteridad, como lo que da a pensar. Marcas en mi de un aprendizaje que estoy comenzando con todo mi cuerpo, mi encuentro vital con algo otro que pude desbloquear muchas de mis antigas creencias y definiciones del mundo.

\section{BIBLIOGRAFIA}

AlOISIO, Victoria. Co-Mediacion. Buenos aires: Editora Ad-Hoc, 1997.

MOORE, Chistopher W. O Processo de mediação. Porto Alegre: Editora Artmed, 1998.

MULDOON, Brian. El corazón del conflicto. Barcelona: Editora Piados, 1998.

GARCÉS, Jose Maria Rossani.(org.) A Arbitragem na era da Globalização. Rio de Janeiro: Editora Forense, 1999.

SIX, François Jean. Dinámica de la mediación. Barcelona: Editora Piados, 1997.

FOLBERT, Jay e outro. Mediación. México: Editora Limusa, 1996.

WARAT, Luis Alberto. (org.) Em nome do acordo. Buenos Aires: Editora Almed, 1999. 